\title{
Effect of ion drift on shock waves in an un- magnetized multi-ion plasma
}

\begin{abstract}
We investigate the effect of the drift velocity of lighter ions on shock waves in an unmagnetized multi-component plasma. Solar origin hot electrons and lighter ions, positively and negatively charged heavier ions and cometary origin colder electrons form the multicomponent plasma. Using the reductive perturbation technique, we have derived the KdVB (Korteweg-deVries-Burgers) equation and its shock wave solution is plotted for different plasma parameters relevant to comet Halley. We find that the strength of the shock profile decreases with increasing drift velocities of the lighter ions and kappa indices of the two components of electrons. On the other hand, the amplitude of the shock increases with increasing kinematic viscosities and densities of the lighter ions. Also, different coefficients of the KdVB equation are strongly affected by the drift velocities of the lighter ions.
\end{abstract}

Keywords: electrons of solar and cometary origin, kappa distribution functions, lighter ions with drift, KdVB equation, shock waves
Volume I Issue 5 - 2018

\author{
Sijo S, ' Anu V, ${ }^{2}$ Neethu TW, ${ }^{3}$ Manesh M, ${ }^{4}$ G \\ Sreekala, ${ }^{5}$ Venugopal $C^{6}$ \\ 'Department of Physics, St. Berchman's College, Changanassery, \\ India \\ ${ }^{2-6}$ School of Pure and Applied Physics, Mahatma Gandhi \\ University, India
}

Correspondence: Venugopal C, School of Pure and Applied Physics, Mahatma Gandhi University, Kottayam, Kerala, India, Email cvgmgphys@yahoo.co.in

Received: June 22, 2018 | Published: September 07, 2018

\section{Introduction}

During the last few decades, multi-component/dusty plasmas have received considerable attention from researchers due to their omnipresence in astrophysical, space and laboratory plasmas. For example, dusty plasmas occur in planetary atmospheres and rings ${ }^{1,2}$ and comets; ${ }^{3}$ nearer Earth they occur in the Earth's mesosphere ${ }^{4}$ and ionosphere ${ }^{5}$ and also in laboratory plasmas used for the formation of 'plasma crystals' ${ }^{6}$ and fusion devices. ${ }^{7}$ The addition of dust to plasmas increases their complexity due to the excitation of different eigen modes like Dust Acoustic Waves (DAWs), ${ }^{8}$ Dust Ion Acoustic Waves (DIAWs), ${ }^{9}$ Dust Lattice Waves (DLWs) ${ }^{10}$ etc.

In addition most plasma waves are not linear: hence a study of nonlinearity, along with dispersion and dissipation, becomes important in the investigations of different plasmas. Among nonlinear phenomena, solitons, shocks and double layers are widely seen in different space environments. ${ }^{11}$ Nonlinearity, along with dispersion, results in the formation of soliton structures with spectacular stability; a plasma supports shock waves if in addition, dissipative effects are also present. The KdVB (Korteweg-deVries-Burgers) equation supports shock waves, with dissipation occurring due to a variety of reasons like wave-particle interactions, turbulence, dust charge, streaming of ions, etc.

A cometary plasma is a true multi-ion plasma composed of species of both solar and cometary origin. Cometary activity begins when it is approaching the Sun: dissociation of water molecules liberates positively charged oxygen and hydrogen and associated photoelectrons; this is in addition to hydrogen and electrons of solar origin. ${ }^{12}$ Other ions observed in a cometary environment include $\mathrm{He}^{+}, \mathrm{He}^{2+}$ $\mathrm{H}_{2}^{+}, \mathrm{OH}^{+}, \mathrm{C}^{+}, \mathrm{H}_{2} \mathrm{O}^{+}, \mathrm{H}_{3} \mathrm{O}^{+}, \mathrm{CO}^{+}$and $\mathrm{S}^{+} .{ }^{13,14}$ Investigations have also revealed the presence of ions of mass $>12 \mathrm{amu},{ }^{15}$ multiply charged ions like $\mathrm{O}^{3+}$ and $\mathrm{O}^{7+}$ and $\mathrm{O}^{8+}$ have been observed ${ }^{16,17}$ at comet McNaughtHartley by the spacecrafts Ulysses and Chandra respectively. Thus oxygen ions are an important component in the plasma composition of a comet.

In addition to positively charged ions, negatively charged ions have also been found in three extended mass peaks of 7-19, 22-65 and $85-110 \mathrm{amu}$ with energies ranging from $0.03-3.0 \mathrm{keV}$ by the Giotto space-craft at comet Halley. Thus it is now well established that positively and negatively charged ions coexist in different cometary environments. ${ }^{4,18-20}$

The presence of different types of inhomogeneities makes particle distributions deviate from equilibrium; hence one has to consider non-Maxwellian distributions. Vasyliunas ${ }^{21}$ first proposed such a distribution while analyzing solar wind data. This distribution is now known as a "kappa" distribution and many space and astrophysical plasmas are best described by this distribution.

The investigation of nonlinear waves in the presence of different drifting components present in plasmas gave a new dimension to nonlinear plasma wave research. Ghosh et al., ${ }^{22}$ studied small amplitude dust acoustic solitons in a two component dusty plasma consisting of ions and drifting dust grains, applicable to the F-ring of Saturn. In another study, applicable to the F, G and E rings of Saturn, they investigated dust drift incorporating ion inertial effect along with dust charge variation. They concluded that there existed an instability due to free energy of drift motion of dust grains. ${ }^{23}$ In yet an another investigation, it was found that the drift velocity of the ions, along with electron inertia, significantly contributes to the formation of double layers and solitary structures in a plasma. ${ }^{24}$ Chattopadhyay ${ }^{25}$ investigated the effect of ion temperature on ion acoustic solitary waves in a drifting negative plasma. In 2012, Tribeche et al., ${ }^{26}$ studied the effect of drift of dust grains on arbitrary amplitude dust acoustic double layers in a warm dusty plasma with two temperature thermal ions and superthermal electrons. In a study related to ion acoustic double layers in magnetospheric and auroral plasmas, multi-drifting components were considered with nonthermal electrons. ${ }^{27}$

There are extensive studies on shock waves in the presence of kappa distributed electrons and ions. ${ }^{28-33}$ In plasmas with super thermal electrons, it was found that spectral index changes the amplitude of the dust acoustic shock waves significantly. ${ }^{28}$ In an electron-positronion (e-p-i) plasma, applicable to pulsar magnetospheres, the effect of plasma parameters on the strength and steepness of the shock structure was investigated, with electrons and protons being described by kappa distributions. ${ }^{29}$ In a plasma with a beam, it was noticed that both the amplitude and steepness of the ion-acoustic shock wave accrued, as the spectral index of the superthermal electrons and concentration of impinging positron beam were enhanced. This can also be applied 
to laboratory beam plasma interaction experiments and space and astrophysical plasmas. ${ }^{30}$ In an investigation of dust acoustic shock waves in a strongly coupled un-magnetized dusty plasma with kappa described ions, it was found that, the coefficients of KdVBurger's equation were significantly modified. ${ }^{31}$ Later, Kourakis et al., ${ }^{32}$ discussed the role of superthermality on the characteristics of electrostatic plasma waves. In a numerical and analytical study of a plasma composed of inertial ions, kappa distributed electrons of two temperatures and negatively charged immobile dust grains, it was seen that the effect of superthermaility significantly modifies the basic features of dust ion acoustic shock waves. ${ }^{33}$

There are a number of observations showing the existence of nonlinear waves at different comets. ${ }^{34-39}$ Cometary missions Giotto and Vega-1 found structures of sub and bow shocks at comet Halley. ${ }^{40}$ Kennel et al., ${ }^{41}$ discussed different plasma waves in the shock interaction regions at comet Giacobini-Zinner. Also, recently nonlinear waves like solitons, etc were found at the same comet. ${ }^{42}$

Thus for reasons given above, we are interested in studying the effect of streaming lighter ions on shock waves in a five component plasma: a pair of oppositely charged heavier ions, streaming lighter ions and two components of electrons.

\section{Basic equations}

As mentioned above we are interested in studying the effect of streaming lighter ions on shock waves in five component plasma. The five components that compose our plasma are a pair of oppositely charged heavier ions, drifting lighter ions and two components of kappa described electrons.

The electrons of both solar and cometary origin are thus described as

$$
n_{s}=n_{s 0}\left[1-\frac{e_{s} \psi}{k_{B} T_{s}\left(\kappa_{s}-3 / 2\right)}\right]^{-\kappa_{s}+1 / 2}
$$

where $n_{S}$ and $n_{S} 0$ are respectively the number and equilibrium values of densities of species ' $\mathrm{s}$ ' ( $\mathrm{s}=$ ce for cometary electrons and $\mathrm{s}$ $=$ he for hot solar electrons), $e_{s}$ is the charge, $k_{B}$ is the Boltzmann's constant, $T_{s}$, the temperature, $\psi$, the potential. $K_{S}$ is the spectral index of species ' $\mathrm{s}$ '.

The fluid equation of continuity governs the dynamics of the plasma components and is given by

$$
\frac{\partial n_{j}}{\partial t}+\frac{\partial}{\partial x} \cdot\left(\mathrm{n}_{j} \mathrm{u}_{j}\right)=0 ; \quad j=i, 1 \text { and } 2
$$
below

The lighter ion (heavier ion (dust)) equations of motion are given

$$
\begin{gathered}
\frac{\partial u_{i}}{\partial t}+\left(\mathrm{u}_{i} \cdot \frac{\partial}{\partial x}\right) \mathrm{u}_{i}=-\alpha_{i} \beta_{i} \frac{\partial \psi}{\partial x}+\eta \frac{\partial^{2} u_{i}}{\partial x^{2}} \\
\frac{\partial u_{1}}{\partial t}+\left(\mathrm{u}_{1} \cdot \frac{\partial}{\partial x}\right) \mathrm{u}_{1}=\frac{\partial \psi}{\partial x} \\
\frac{\partial u_{2}}{\partial t}+\left(\mathrm{u}_{2} \cdot \frac{\partial}{\partial x}\right) \mathrm{u}_{2}=-\alpha_{2} \beta_{2} \frac{\partial \psi}{\partial x}
\end{gathered}
$$

The above set is completed by the Poisson's equation, given by

$$
\begin{aligned}
\frac{\partial^{2} \psi}{\partial x^{2}} & =n_{1}-\left(1-z_{i} \mu_{i}+\mu_{c e}+\mu_{s e}\right) n_{2}-\left(1-z_{2} \mu_{2}+\mu_{c e}+\mu_{s e}\right) n_{i} \\
& +\mu_{c e}\left(1-\frac{\psi}{\sigma_{c e}\left(\kappa_{c e}-3 / 2\right)}\right)^{-\kappa_{c e}+1 / 2}+\mu_{s e}\left(1-\frac{\psi}{\sigma_{s e}\left(\kappa_{s e}-3 / 2\right)}\right)^{-\kappa_{s e}+1 / 2}
\end{aligned}
$$

The above equations (2)-(6) are made dimensionless as follows: the densities and speeds of the plasma species are, respectively, normalized by corresponding equilibrium values of densities $n_{s 0}$ and $\left(\frac{z_{1} k_{B} T_{1}}{m_{1}}\right)^{1 / 2}$. The space (x) and time (t) variables are normalized by the Debye length $\left(\lambda_{D 1}=\left(\frac{z_{1} k_{B} T_{1}}{4 \pi z_{1}^{2} e^{2} n_{10}}\right)^{1 / 2}\right)$ and the inverse of the plasma frequency $\left(\left(\omega_{1}\right)^{-1}\right)$ respectively. The kinematic viscosity of the lighter ions is normalized by $\omega_{1} \lambda_{D 1}^{2}$ and potential $\psi$ by $\frac{k_{B} T_{e}}{e}$. Also $\quad \alpha_{i}=\frac{z_{i}}{z_{1}}, \quad \alpha_{2}=\frac{z_{2}}{z_{1}}, \beta_{i}=\frac{m_{1}}{m_{i}}, \beta_{2}=\frac{m_{1}}{m_{2}}, \mu_{s}=\frac{n_{s 0}}{z_{1} n_{10}}$ and $\sigma_{s}=\frac{T_{s}}{T_{1}}$; where $m_{i}, m_{1}$ and $m_{2}$ and $z_{i}, z_{1}$ and $z_{2}$ are respectively the masses and charges of the lighter ions, negatively and positively charged heavier ions respectively. $T_{s}$ and $T_{1}$ respectively represent the temperatures of the species 's' and negatively charged heavier ions.

To derive the KdVB equation, we use the transformations:

$$
\xi=\varepsilon^{1 / 2}(x-\lambda t) \text { and } \tau=\varepsilon^{3 / 2} t
$$

Therefore

$$
\frac{\partial}{\partial x}=\varepsilon^{1 / 2} \frac{\partial}{\partial \xi} \text { and } \frac{\partial}{\partial t}=\varepsilon^{3 / 2} \frac{\partial}{\partial \tau}-\varepsilon^{1 / 2} \lambda \frac{\partial}{\partial \xi}
$$

The different physical quantities in the equations (1-6) can be expressed asymptotically as a power series about their equilibrium values as:

$$
\begin{gathered}
n_{i, 1,2}=1+\varepsilon n_{i, 1,2}^{(1)}+\varepsilon^{2} n_{i, 1,2}^{(2)}+\ldots \ldots \ldots \\
u_{i, x}=u_{0}+\varepsilon u_{i, x}^{(1)}+\varepsilon^{2} u_{i, x}^{(2)}+\ldots \ldots \ldots . \\
u_{1,2}=\quad \varepsilon u_{1,2}^{(1)}+\varepsilon^{2} u_{1,2}^{(2)}+\ldots \ldots \ldots . \\
\psi=\varepsilon \psi^{(1)}+\varepsilon^{2} \psi^{(2)}+\ldots \ldots \ldots \\
\eta=\varepsilon^{1 / 2} \eta_{i 0}
\end{gathered}
$$

Using transformations (8) and equations (9-13) in equations (1-6) and equating different

$$
\begin{gathered}
n_{i}^{(1)}=\frac{\alpha_{i} \beta_{i}}{\left(\lambda-u_{0}\right)^{2}} \psi^{(1)}, n_{1}^{(1)}=\frac{-\psi^{(1)}}{\lambda^{2}}, n_{2}^{(1)}=\frac{\alpha_{2} \beta_{2}}{\lambda^{2}} \psi^{(1)}, u_{i}^{(1)}=\frac{\alpha_{i} \beta_{i}}{\left(\lambda-u_{0}\right)} \psi^{(1)}, \\
u_{1}^{(1)}=\frac{-\psi^{(1)}}{\lambda}, u_{2}^{(1)}=\frac{\alpha_{2} \beta_{2}}{\lambda} \psi^{(1)}
\end{gathered}
$$

Equating terms of powers of $\varepsilon$ and using (14) in Poisson's equation, we get an expression for the phase velocity of the wave as:

$$
\begin{aligned}
& \left(\frac{\mu_{c e}}{\sigma_{c e}} \frac{\left(\kappa_{c e}-1 / 2\right)}{\left(\kappa_{c e}-3 / 2\right)}+\frac{\mu_{s e}}{\sigma_{s e}} \frac{\left(\kappa_{s e}-1 / 2\right)}{\left(\kappa_{s e}-3 / 2\right)}\right)\left(\lambda^{2}\left(\lambda-u_{0}\right)^{2}\right) \\
& \quad-\left(1-z_{2} \mu_{2}+\mu_{c e}+\mu_{s e}\right) \alpha_{i} \beta_{i} \lambda^{2}-\left(1+\left(1-z_{i} \mu_{i}+\mu_{c e}+\mu_{s e}\right) \alpha_{2} \beta_{2}\right)\left(\lambda-u_{0}\right)^{2}=0
\end{aligned}
$$

Equating terms of power $\varepsilon^{5 / 2}$ and using (14), we get

$$
\begin{aligned}
\frac{\partial n_{i}^{(2)}}{\partial \xi}= & \frac{2 \alpha_{i} \beta_{i}}{\left(\lambda-u_{0}\right)^{3}} \frac{\partial \psi^{(1)}}{\partial \tau}+\frac{3 \alpha_{i}^{2} \beta_{i}^{2}}{\left(\lambda-u_{0}\right)^{4}} \psi^{(1)} \frac{\partial \psi^{(1)}}{\partial \xi}+\frac{\alpha_{i} \beta_{i}}{\left(\lambda-u_{0}\right)^{2}} \frac{\partial \psi^{(2)}}{\partial \xi} \\
& -\frac{\alpha_{i} \beta_{i}}{\left(\lambda-u_{0}\right)^{3}} \eta_{i 0} \frac{\partial^{2} \psi^{(1)}}{\partial \xi^{2}}
\end{aligned}
$$




$$
\begin{gathered}
\frac{\partial n_{1}^{(2)}}{\partial \xi}=\frac{-2}{\lambda^{3}} \frac{\partial \psi^{(1)}}{\partial \tau}+\frac{3}{\lambda^{4}} \psi^{(1)} \frac{\partial \psi^{(1)}}{\partial \xi}-\frac{1}{\lambda^{2}} \frac{\partial \psi^{(2)}}{\partial \xi} \\
\frac{\partial n_{2}^{(2)}}{\partial \xi}=\frac{2 \alpha_{2} \beta_{2}}{\lambda^{3}} \frac{\partial \psi^{(1)}}{\partial \tau}+\frac{3 \alpha_{2}^{2} \beta_{2}^{2}}{\lambda^{4}} \psi^{(1)} \frac{\partial \psi^{(1)}}{\partial \xi}+\frac{\alpha_{2} \beta_{2}}{\lambda^{2}} \frac{\partial \psi^{(2)}}{\partial \xi}
\end{gathered}
$$

Equating $\varepsilon^{2}$ terms from Poisson's equation, taking the derivative and using equations (14-18), we arrive at the KdVB equation as

$$
\begin{aligned}
& A \frac{\partial \psi^{(1)}}{\partial \tau}+\psi^{(1)} \frac{\partial \psi^{(1)}}{\partial \xi}+B \frac{\partial^{3} \psi^{(1)}}{\partial \xi^{3}}-C \frac{\partial^{2} \psi^{(1)}}{\partial \xi^{2}}=0 \\
& A=\frac{A_{0}}{A_{1}}, B=\frac{1}{A_{1}} \text { and } C=\frac{A_{2}}{A_{1}}
\end{aligned}
$$

Where

$$
\begin{gathered}
A_{0}=\frac{2}{\lambda^{3}}+\frac{2 \alpha_{2} \beta_{2}}{\lambda^{3}}\left(1-z_{i} \mu_{i}+\mu_{c e}+\mu_{s e}\right)+\frac{2 \alpha_{i} \beta_{i}}{\left(\lambda-u_{0}\right)^{3}}\left(1-z_{2} \mu_{2}+\mu_{c e}+\mu_{s e}\right) \\
A_{1}=\frac{-3}{\lambda^{4}}+\frac{3 \alpha_{2}^{2} \beta_{2}^{2}}{\lambda^{4}}\left(1-z_{i} \mu_{i}+\mu_{c e}+\mu_{s e}\right)+\frac{3 \alpha_{i}^{2} \beta_{i}^{2}}{\left(\lambda-u_{0}\right)^{4}}\left(1-z_{2} \mu_{2}+\mu_{c e}+\mu_{s e}\right) \\
-\left(\frac{\mu_{c e}\left(\kappa_{c e}-1 / 2\right)\left(\kappa_{c e}+1 / 2\right)}{\sigma_{c e}^{2}\left(\kappa_{c e}-3 / 2\right)^{2}}+\frac{\mu_{s e}\left(\kappa_{s e}-1 / 2\right)\left(\kappa_{s e}+1 / 2\right)}{\sigma_{s e}^{2}\left(\kappa_{s e}-3 / 2\right)^{2}}\right)
\end{gathered}
$$$$
\text { and } A_{2}=\frac{\alpha_{i} \beta_{i} \eta_{i 0}}{\left(\lambda-u_{0}\right)^{3}}\left(1-z_{2} \mu_{2}+\mu_{c e}+\mu_{s e}\right)
$$

\section{Solution of KdVB equation}

To find the solution to (19), we use the transformation $\chi=f(\xi-V \tau)$ of the co-moving frame with speed $\mathrm{V}$. A convenient method to solve the KdVB equation is the "tanh method". ${ }^{43,44}$ Using boundary conditions $\psi^{(1)}, \frac{\partial \psi^{(1)}}{\partial \chi}, \frac{\partial^{2} \psi^{(1)}}{\partial \chi^{2}}, \frac{\partial^{3} \psi^{(1)}}{\partial \chi^{3}} \rightarrow 0$, as $\chi \rightarrow \infty$ for a localized solution, we can write (19) as

$$
-A V \frac{\partial \psi^{(1)}}{\partial \tau}+\psi^{(1)} \frac{\partial \psi^{(1)}}{\partial \xi}+B \frac{\partial^{3} \psi^{(1)}}{\partial \xi^{3}}-C \frac{\partial^{2} \psi^{(1)}}{\partial \xi^{2}}=0
$$

Using the transformation $\psi^{(1)}(\alpha)=\sum_{i=0}^{n} a_{i} \alpha^{i}, \quad \alpha=\tanh \chi$, we can arrive at the solution as

$\psi^{(1)}=A V+8 B k^{2}+\frac{C^{2}}{25 B}-12 k^{2} \tanh ^{2}[k(\xi-V \tau)]-\frac{12}{5} k C[1-\tanh [k(\xi-V \tau)]]$

The speed of the co-moving frame is related to the coefficients of the KdVB equation as $V=\frac{100 B^{2} k^{2}-C^{2}+60 k B C}{25 A B}$ where $k=\frac{ \pm C}{10 B}$, obtained from the boundary conditions.

\section{Results}

We have derived equations applicable to any multi-ion/dusty plasma environment; the figures, are, however, plotted using parameters relevant to comet Halley. The majority lighter ion density was set at $4.95 \mathrm{~cm}^{-3}$, and their temperature at $T_{i}=8 \times 10^{4} \mathrm{~K}$ and solar electron temperature at ${ }^{12} T_{h e}=2 \times 10^{5} \mathrm{~K}$. The negatively charged heavier ions (oxygen) was detected at an peak energy of $\sim 1 \mathrm{eV}$ with densities ${ }^{18} \leq 1 \mathrm{~cm}^{-3}$. The negatively and positively charged heavier ion densities are therefore $n_{1}=0.05 \mathrm{~cm}^{-3}$ and $n_{2}=0.5 \mathrm{~cm}^{-3}$ respectively at a temperature ${ }^{12,18} 1.16 \times 10^{4} \mathrm{~K}$.

Figure 1 depicts the effect of the drift velocities of the lighter ions on the shock profile. The parameters used for the figure are:

$n_{i 0}=4.95 \mathrm{~cm}^{-3}, \quad n_{10}=0.05 \mathrm{~cm}^{-3}, \quad n_{20}=0.5 \mathrm{~cm}^{-3}, \quad T_{c e}=2 \times 10^{4} \mathrm{~K}$, $T_{h e}=2 \times 10^{5} \mathrm{~K}, \quad T_{1}=1.16 \times 10^{4} \mathrm{~K}, z_{1}=2, z_{2}=4, \quad B_{0}=8 \times 10^{-5} \mathrm{G}$, $\kappa_{c e}=\kappa_{h e}=3$ and $\eta_{i 0}=0.5$. The upper plot (blue in color) depicts the shock profile without a drift velocity for the lighter ions, as discussed by Manesh et al., ${ }^{45}$ the shock is produced by the effects of heavier positive and negative ions. The profile in the middle (green in color) is for a drift velocity of lighter ions $u_{0}=0.2$ and lower (red in color) is for $u_{0}=0.4$. From the plots it is seen that as drift velocities of the lighter ions increase, the amplitude of the shock profile decreases.

The shock profiles, for different kappa indices of both solar and cometary electrons, are depicted in Figure 2. The lower plot is for $\kappa_{c e}=\kappa_{h e}=3$ for $u_{0}=0.3$ (continuous blue line) and $u_{0}=0$ (blue dashed line); the middle plot is for $\kappa_{c e}=\kappa_{h e}=5$ for $u_{0}=0.3$ (continuous green line) and $u_{0}=0$ (green dashed line) while the upper plot is for $\kappa_{c e}=\kappa_{h e}=10$ for $u_{0}=0.3$ (continuous red line) and $u_{0}=0$ (dashed red line). The other parameters are the same as in figure 1 . We find that the strengths of the shock profiles decrease as the kappa values increase, irrespective of the drift velocities of the lighter ions. Lower values of kappa indices indicate the presence of more superthermal particles in the plasma; these superthermal particles thus support larger shock profiles. Also, as the plasma approaches a Maxwellian distribution, the effect of the drift velocity diminishes. In addition, the strength of the shock profile decreases for non-zero values of drift velocity as compared to the profile for a zero drift velocity of the lighter ions; in agreement with Figure 1.

Next, Figure 3 shows the variation of shock profiles in the plasma for different values of kinematic viscosity with drift velocity of lighter ions included $\left(u_{0}=0.1\right)$. The upper curve (blue in color) is for kinematic viscosity $\eta_{i 0}=0.1$, the middle curve (green in color) is for $\eta_{i 0}=0.3$ and the lower curve (red in color) is for $\eta_{i 0}=0.5$. The other parameters are the same as in Figure 1. From the figure it is seen that the size of the shock profile increases with increasing values of kinematic viscosities of the lighter ions.

Figure 4 is a plot of shock profiles for different densities and drift velocities of the lighter ions. The upper plot (blue in color) is for $n_{i 0}=3$, middle one (green in color) for $n_{i 0}=4$ and lower plot (red in color) is for $n_{i 0}=5$ with drift velocities of lighter ions as $u_{0}=0.5$ and $u_{0}=0.1$ (denoted respectively by continuous and dashed lines). The other parameters are the same as in figure 1. From the plots, it is evident that the amplitude of the shock profile increases as the density of lighter ions increases.

Figure 5 depicts values of coefficient $\mathrm{A}$ as a function of the drift velocities of the lighter ions. The other parameters for the plot remain the same as in Figure 1. From the plot, it is seen that value of coefficient increases exponentially as the drift velocity increases.

Finally, Figure 6 illustrates the values of the coefficient B as a 
function of the drift velocities of the lighter ions. The other parameters for the plot remain the same as in Figure 1. It is obvious from the plot that the variation of $\mathrm{B}$, with the drift velocity of the lighter ions, is almost linear.

Figure I Plot of shock profiles for different drift velocities of the lighter ions.

Figure 2 Plot of shock profiles for different kappa indices with and without

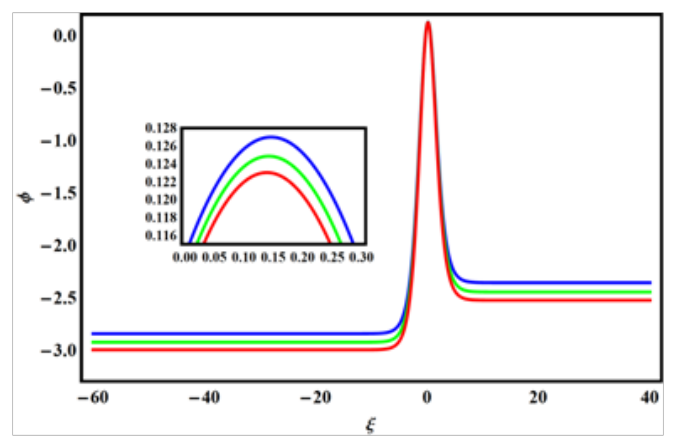

drift for the lighter ions.

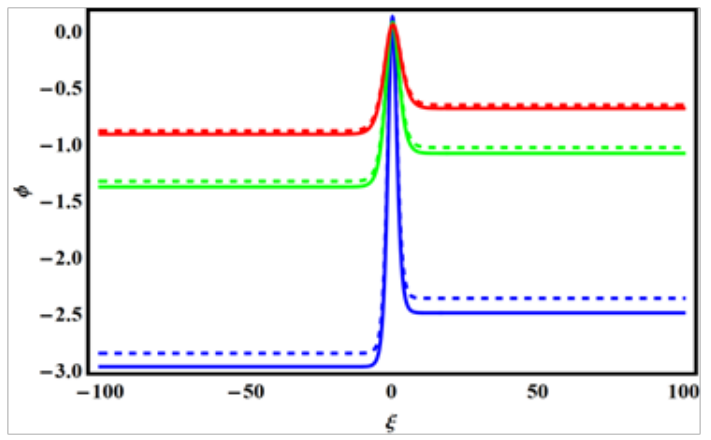

Figure 3 Plot of Shock profiles for different values of kinematic viscosity $\eta_{i 0}$ with a drift velocity $\left(u_{0}=0.1\right)$ of lighter ions.

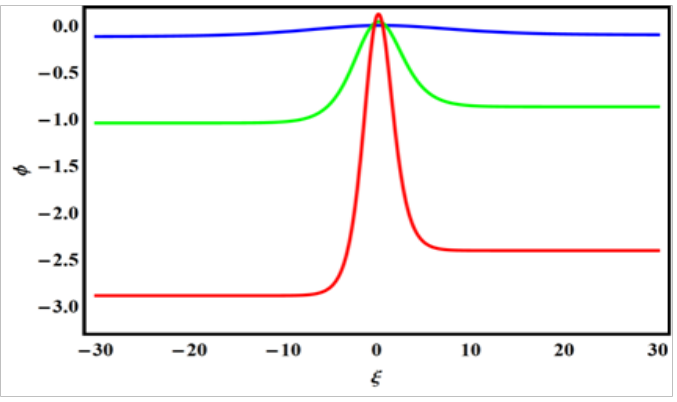

Figure 4 Plot of shock profiles for different densities and drift velocities of lighter ions.

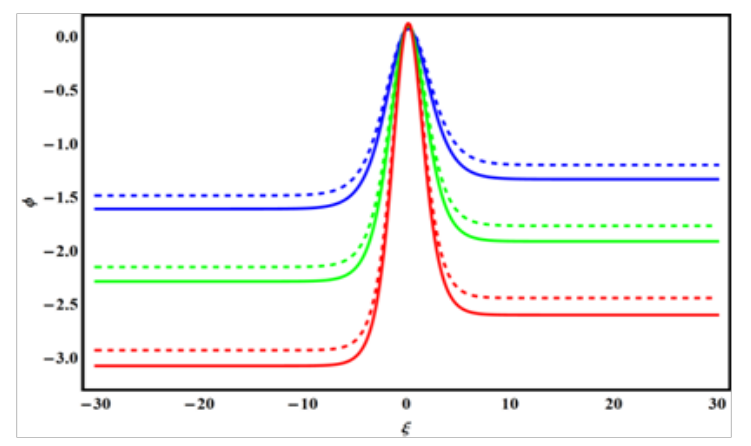

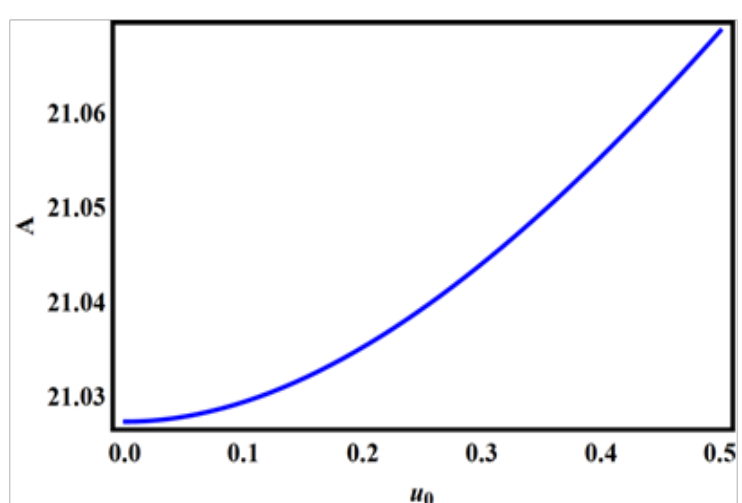

Figure 5 Plot of coefficient $A$ for different drift velocities of lighter ions.

Figure 6 Plot of coefficient B for different drift velocities of lighter ions.

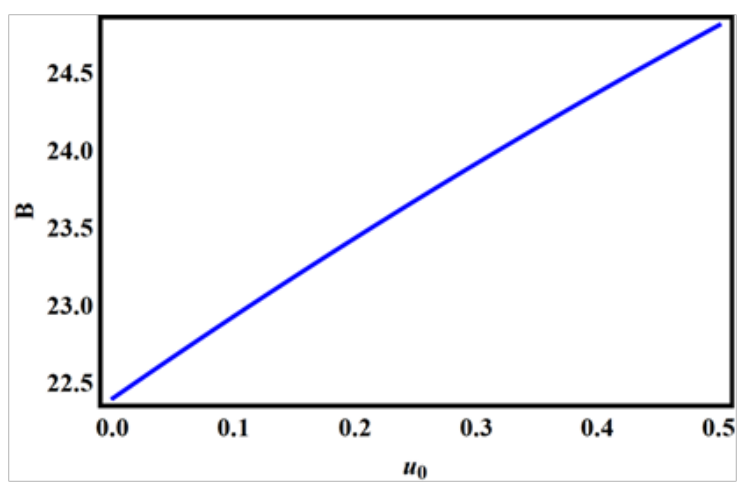

Figure 1 and Figure 2 given above reveal that the shock amplitudes decrease with increasing streaming velocities of the lighter ions. For the given plasma system some of the plasma instabilities, whose growth rates are a function of the streaming velocity, that can be excited are the two stream instability, the ion acoustic instability, etc. Thus the decrease in shock amplitude could be due to a diversion of the streaming energy to excite these instabilities. At this point it may be noted that the existence of both these waves were noticed in numerical simulation studies of shock dynamical behavior. ${ }^{46}$ A superthermal distribution tends to a Maxwellian distribution when the spectral index $\kappa->\infty$, thus damping the wave; a larger streaming velocity is now required to drive the instability. Hence the shock amplitude decreases when the spectral index $\kappa->\infty$. Such a decrease in the amplitude of the shock wave with spectral index was also found in a study of ion acoustic shock waves in a dissipative plasma with superthermal electrons and positrons. ${ }^{47}$ Figure 3 reveals that the amplitude of the shock wave increases with increasing lighter ion kinematic viscosity. This is due to the increased dissipation in the system and has been reported earlier in an electron-positron-ion plasma. ${ }^{48}$ Keeping the ion kinematic viscosity a constant and increasing the lighter ion density again increases the dissipation and hence explains the results of figure. Finally, the equations describing the system can be made applicable to any multi-ion environment where both polarities of heavier ions are present along with superthermal electrons and drift velocity of lighter ions.

\section{Conclusion}

The KdVB equation has been derived in an un-magnetized plasma composed of kappa function described electrons (of both cometary and solar origin), positively and negatively charged heavier ions and lighter ions with a streaming velocity. The shock profiles have been studied for various parameters like kinematic viscosities of the lighter 
ions, drift velocities and kappa values. It is seen that the drift velocity has a strong influence on the shock wave: its amplitude decreases as the drift velocities of the lighter ions increases. Also, the amplitude of the shock profiles increases with a decrease in the kappa indices of the electrons and increases with an increase in the densities of the lighter ions. That is, increasing the number of superthermal electrons and lighter ions supports high amplitude shock waves in the plasma. In other words, increasing the number density of the dissipative plasma species increases the amplitude of the shock waves, irrespective of their polarity. Also, different coefficients of the KdVB equation are strongly affected by the drift velocities of the lighter ions.

\section{Acknowledgements}

We thank the reviewer for the very useful comments which have added immensely to the scientific content of the paper.

\section{Conflict of interest}

The author declares that there is no conflict of interest.

\section{References}

1. Goertz CK. Dusty Plasmas in the Solar System. Rev Geophys. 1989;27(2):271-292.

2. Northrop TG. Dusty Plasmas. Phys Scripta. 1992;45(5):475-490

3. Egrand C, Duprant J, Dartois E, et al. Variations in cometary dust composition from Giotto to Rosetta, clues to their formation mechanisms. MNRAS. 2016;462(Suppl 1):323-S330.

4. Havnes O, Troim J, Blix T, et al. First detection of charged particles in the Earth's mesosphere. J Geophys Res. 1996;101(A5):10839-10847.

5. Dubinskii A, Popel SI. Formation and evolution of dusty plasmas in the ionosphere. JETP Letters. 2012;96(1):21-26

6. Thomas H, Morfill GE, Demmel V, et al. Plasma crystal: coulomb crystallization in a dusty plasma. Phys Rev Lett. 1994;73(5);652-655.

7. Winter J. Dust in Fusion Devices. Plas Phys Contr Fus. 2004;12B:583

8. Rao NN, Shukla PK, Yu, et al. Dust-acoustic waves in dusty plasmas. Planet Space Sci. 1990;38(4):543-546.

9. Shukla PK, Silin VP. Dust ion-acoustic wave. Physica Scr. 1992;45(5):508.

10. Melandso M. Lattice Waves in dust plasma crystals. Phys Plasmas. 1996;3(11):3890-3901.

11. Shukla PK, Mamun AA. Solitons, shocks and vortices in dusty plasmas New J Phys. 2003;5(1):17-37.

12. Brinca AL, Tsurutani BT. Unusual characteristics of the electromagnetic waves excited by cometary newborn ions with large perpendicular energies. Astron \& Astrophys. 1987;187:311-319.

13. Balsiger H, Altwegg K, Bühler F, et al. Ion composition and dynamics at comet Halley. Nature. 1986;321:330-334.

14. Rubin M, Hansen KC, Gombosi TI, et al. Ion composition and chemistry in the coma of Comet $1 \mathrm{P} / \mathrm{Halley}-\mathrm{A}$ comparison between Giotto's Ion Mass Spectrometer and our ion-chemical network. Icarus. 2009;199(2):505-519.

15. Ipavich F, Galvin A, Gloeckler G, et al. Comet Giacobini-Zinner: In Situ observations of energetic heavy ions. Science. 1986;232(4748):366-369.

16. Neugebauer M, Gloeckler G, Gosling JT, et al. Encounter of the
Ulysses spacecraft with the ion tail of comet McNaught. Astrophy $J$ 2007;667(2):1262-1266.

17. Kharchenko V, Rigazio M, Dalgarno A, et al. Charge abundances of the solar wind ions inferred from cometary X-ray spectra. Astrophys J Lett. 2003;585:73-L75.

18. Chaizy P, Reme H,Sauvaud, JA, et al. Negative ions in the coma of comet Halley. Nature. 1991;349:393-396.

19. Ellis TA, Neff JS. Numerical simulation of the emission and motion of neutral and charged dust from P/Halley. Icarus. 1991;91(2):280-296.

20. Chow VW, Mendis DA, Rosenberg M. Role of grain size and particle velocity distribution in secondary electron emission in space plasmas. $J$ Geophys Res. 1993;98(A11):19065-19076.

21. Vasyliunas VM. A survey of low-energy electrons in the evening sector of the magnetosphere with OGO 1 and OGO 3. J Geophys Res. 1968;73(9):2839-2884

22. Ghosh S, Sarkar S, Khan M. Effect of finite ion inertia and dust drift on small amplitude dust acoustic soliton. Planet. Space Sci. 2000;48:609614.

23. Ghosh S, Chaudhuri TK, Sarkar S, et al. Small amplitude nonlinear dust acoustic wave propagation in Saturn's F, G and E rings. Astrophys Space Sci. 2001;278(4):465-477.

24. Paul SN, Chattopadhyay S, Bhattacharya SK, et al. On the study of ionacoustic solitary waves and double-layers in a drift multi-component plasma with electron-inertia. Pramana. 2003;60(6):1217-1233.

25. Chattopadhyay S. Effect of ionic temperatures on ion-acoustic solitary waves in a drift negative-ion plasma with single temperature electron. Fizika A-J Exper Theor Phys-Zagreb. 2010;19:31-46.

26. Tribeche M, Younsi S, Zerguini TH. Arbitrary amplitude dust-acoustic double-layers in a warm dusty plasma with suprathermal electrons, twotemperature thermal ions, and drifting dust grains. Astrophys Space Sci. 2012;339(2):243-247.

27. Ghosh B, Paul SN, Das C, et al. Electrostatic double layers in a multicomponent drifting plasma having nonthermal electrons. Braz J Phys. 2013;43(1-2):28-33.

28. Kundu SK, Ghosh DK, Chatterjee P, et al. Shock waves in a dusty plasma with positive and negative dust, where electrons are superthermally distributed. Bulg J Phys. 2011;38(2011):409-419.

29. Shah A, Saeed R. Nonlinear Korteweg-de Vries-Burger equation for ion-acoustic shock waves in the presence of kappa distributed electrons and positrons. Plasma Phys Control Fus. 2011;53(9):095006

30. Shah A, Mahmood S, Haque Q. Ion acoustic shock waves in presence of superthermal electrons and interaction of classical positron beam. Phys Plasmas, 2012;19:032302

31. Pakzad HR. Dust acoustic shock waves in strongly coupled dusty plasmas with kappa-distributed ions. Indian J Phys. 2012;86(8):743-747.

32. Kourakis I, Sultana S, Hellberg MA. Dynamical characteristics of solitary waves, shocks and envelope modes in kappa-distributed non-thermal plasmas: an overview. Plasma Phys Control Fus. 2012:54(12):124001124007

33. Alam MS, Masud MM, Mamun AA. Effects of bi-kappa distributed electrons on dust-ion-acoustic shock waves in dusty superthermal plasmas. Chinese Phys. B 2013;22:115202.

34. Hyder CL, Brandt JC, Roosen RG. Tail structures far from the head of Comet Kohoutek I. Icarus. 1974;23:601-610.

35. Ershkovich AI. Solar wind interaction with the tail of come 
Kohoutek. Planet. Space Sci. 1976;24(3):287-291.

36. Ershkovich AI, Heller AB. Helical waves in type-1 comet tails. Astrophys. Space Sci. 1977;48:365-377.

37. Hada T, Kennel CF, Buti B. Stationary nonlinear Alfvén waves and solitons. J Geophys Res. 1989;94:65-77.

38. Tsurutani BT, Smith EJ, Matsumoto H, et al. Highly nonlinear magnetic pulses at comet Giacobini-Zinner. Geophys. Res. Lett.1990;17(6):757760 .

39. Kotsarenko NY, Koshevaya SV, Stewart GA, et al. Electrostatic spatially limited solitons in a magnetised dusty plasma. Planet Space Sci. 1998;46(4):429-433.

40. Coates AJ. Heavy ion effects on cometary shocks. Adv Space Res 1995;15(8-9):403-413.

41. Kennel CF, Coroniti FV, Scarf FL, et al. Plasma waves in the shock interaction regions at comet Giacobini-Zinner. Geophys. Res Lett.1986;13(9):921-924.

42. Voelzke MR, Izaguirre LS. Morphological analysis of the tail structures of comet P/Halley 1910 II. Planet Space Sci. 2012;65(1):104-108.
43. Malfliet W. Solitary wave solutions of nonlinear wave equations. American J Phys. 1992;60:650-654.

44. Malfliet W. The tanh method:a tool for solving certain classes of nonlinear evolution and wave equations. J Comput Appl Math. 2004;164-165:529541.

45. Manesh M, Neethu TW, Neethu J, et al. Korteweg-deVries-Burgers (KdVB equation) equation in a five component plasma cometary plasma with kappa described electrons and ions. J Theor Appl Phys. 2015;10(4):280-296

46. Shimada N, Hoshino M. Effect of strong thermalization on shock dynamical behavior. J Geophys Res. 2005;110(2):A02105

47. Pakzad HR. Ion Acoustic shock waves in dissipative plasma with superthermal electrons and positrons. Astrophys Space Sci. 2011;331(1):169-174

48. Ghosh DB, Chatterjee P, Mandal PJ, et al. Nonplanar ion-acoustic shocks in electron-positron-ion plasmas:effect of superthermal electrons. Pramana-J Phys. 2013;81(3):491-501 